\title{
Soluções de Problemas envolvendo Equações Diferenciais Sujeitas a Incertezas
}

F.A. DORINI ${ }^{1}$ Departamento Acadêmico de Matemática, UTFPR - Univ Tecnológica Federal do Paraná, 80230-901 Curitiba, PR, Brasil.

M.C.C. CUNHA2, Departamento de Matemática Aplicada, UNICAMP - Univ Estadual de Campinas, 13083-970 Campinas, SP, Brasil.

S.P. OLIVEIRA ${ }^{3}$, Departamento de Matemática, UFPR - Univ Federal do Paraná, 81531-990 Curitiba, PR, Brasil.

Resumo. Este trabalho objetiva analisar, através de alguns exemplos, a influência de se considerar aleatoriedades na solução de equações diferenciais com dados e/ou parâmetros aleatórios. Um comparativo das médias das soluções das equações estocásticas com as soluções das equações determinísticas simplificadas, nas quais substituímos os parâmetros aleatórios por suas médias, é apresentado. Estes exemplos mostram que a média da solução, que normalmente é uma informação relevante em aplicações, pode ser qualitativamente diferente da aproximação obtida pela solução de uma equação diferencial determinística na qual substituímos os parâmetros aleatórios por suas médias.

Palavras-chave. Equações diferenciais estocásticas, variáveis aleatórias, esperança matemática, função de densidade de probabilidade.

\section{Introdução}

A modelagem de fenômenos físicos através de equações diferenciais cujos dados não são precisamente conhecidos pode ser realizada utilizando modelos probabilísticos (estocásticos) de tais dados. Os modelos probabilísticos compensam a escassez de dados e fornecem margens de erros para estimativas de laboratório. O escoamento em meios porosos se encaixa neste perfil. Propriedades como a porosidade e a condutividade hidráulica são estimadas, por exemplo, por meio de amostras do meio poroso. Resultados experimentais sugerem que a distribuição lognormal representa satisfatoriamente estas propriedades [22]. Quando modelos probabilísticos são acoplados aos parâmetros das equações diferenciais que governam o escoamento de fluidos, o modelo completo passa a ser descrito por equações diferenciais estocásticas.

\footnotetext{
${ }^{1}$ fabio.dorini@gmail.com

${ }^{2}$ cunha@ime.unicamp.br

${ }^{3}$ saulopo@ufpr.br

Este trabalho foi apresentado no XXXIII CNMAC - Congresso Nacional de Matemática Aplicada e Computacional, 2010, Águas de Lindóia, SP, Brasil [6].
} 
$\mathrm{Na}$ tentativa de resolver problemas deste tipo, duas vertentes têm sido motivos de estudos nas últimas décadas. A mais formal segue da interpretação das equações diferenciais estocásticas como equações integrais num sentido mais geral, inicialmente introduzido por Itô, em meados de 1940, e generalizado por outros autores (veja $[7,10,14,19]$, por exemplo). A extensão destas fórmulas às equações diferenciais aparece a partir da década 1960. Por exemplo, em [4] são examinados resultados gerais e recentes da análise funcional de alguns tipos de equações diferenciais estocásticas sob condições de regularidade exigidas dos dados.

$\mathrm{Na}$ outra vertente estão métodos numéricos e técnicas matemáticas cujo objetivo é obter informações práticas sobre a função aleatória (estocástica) que é solução do problema, no sentido probabilístico. Estas informações podem ser a média (esperança matemática), alguns momentos de ordem superior ou, melhor ainda, a função de densidade de probabilidade da solução. O método de Monte Carlo [8] foi o primeiro nesta direção e se tornou conhecido nas simulações da construção das primeiras bombas atômicas quando, também, os primeiros computadores estavam sendo usados na computação científica. A ideia do método é calcular médias, ou outros momentos, usando um grande número de ensaios experimentais, usualmente gerados com sequências pseudoaleatórias. Cada um destes ensaios deve resultar em um problema determinístico cuja solução possa ser calculada. Em outra direção (do ponto de vista teórico), alguns autores procuram equações diferenciais determinísticas cujas soluções podem ser médias, momentos [9, 18, 20, 22], e até mesmo a função de densidade de probabilidade $[11,16]$ da solução.

Este trabalho objetiva analisar, através de alguns exemplos, a influência de se considerar aleatoriedades na solução de equações diferenciais com dados aleatórios. Um estudo comparativo das médias das soluções das equações estocásticas com as soluções das equações determinísticas simplificadas, nas quais substituímos os parâmetros aleatórios por suas médias, é apresentado. Cabe ressaltar que o maior mérito do trabalho é o de ilustrar a importância de se obter as soluções estocásticas das equações, via distribuição ou esperança matemática. Os autores esperam que esse trabalho possa fomentar novas investigações em uma área de importância crescente na modelagem Matemática.

Embora modelos aleatórios discretos sejam importantes em diversas aplicações, os exemplos tratados aqui concentram-se em modelos contínuos. Considere um espaço de probabilidade $(\Omega, \mathcal{F}, \mathcal{P})$ e uma variável aleatória $A: \Omega \rightarrow \mathbf{R}$, com função de densidade de probabilidade $f_{A}$. Sua função de distribuição cumulativa é dada por

$$
F_{A}(a)=\mathcal{P}(\{\omega \in \Omega \mid A(\omega) \leq a\})=\mathcal{P}(A \leq a)=\int_{-\infty}^{a} f_{A}(u) d u .
$$

A média, ou esperança matemática, da variável aleatória $A$ é definida por

$$
\mathrm{E}[A]=\int_{-\infty}^{+\infty} a f_{A}(a) d a .
$$

Nos três problemas seguintes, um ou mais parâmetros das equações diferenciais são variáveis aleatórias. Tendo em vista o propósito de analisar a influência dessas aleatoriedades nas equações, é apresentada, para cada problema, um comparativo 
entre a média da solução estocástica e a solução da equação simplificada, na qual a variável aleatória $A$ é substituída pela sua média, $\mathrm{E}[A]$.

Cabe enfatizar que o uso de variáveis aleatórias para modelar a incerteza nestes problemas difere da abordagem empregada no Cálculo Estocástico Clássico [4, 7, $10,14]$, na qual os dados de entrada são processos idealizados, tais como processos de Wiener e processos de Poisson [20, p. 3]. Isto indica, junto com as técnicas matemáticas ou computacionais empregadas, uma separação entre os escopos da vertente do Cálculo Estocástico Clássico e a vertente adotada no presente trabalho.

\section{Problema de Valor de Contorno Aleatório}

O ponto de partida é o seguinte problema de valor de contorno:

$$
\begin{aligned}
& -\frac{d}{d x}\left(A \frac{d U}{d x}(x)\right)=1, \quad 0<x<1, \\
& U(0)=U(1)=0,
\end{aligned}
$$

em que $A$ é uma variável aleatória que assume apenas valores positivos, isto é, $\mathcal{P}(A \leq 0)=0$. Este problema pode ser visto como um modelo rudimentar de escoamentos em meios porosos, em que $U$ corresponde à carga hidráulica em um reservatório cuja permeabilidade hidráulica é representada pelo parâmetro aleatório $A$ [1]. A aleatoriedade de $A$ implica que a solução do problema aleatório acima é uma função aleatória (ou, mais precisamente, um processo estocástico [15]). De fato, para cada realização $A(\omega)$, de $A$, considere o problema determinístico:

$$
\left\{\begin{array}{l}
-\frac{d}{d x}\left(A(\omega) \frac{d U}{d x}(x, \omega)\right)=1, \quad 0<x<1, \\
U(0, \omega)=U(1, \omega)=0,
\end{array}\right.
$$

cuja solução é obtida integrando duas vezes os lados da equação e impondo as condições de contorno:

$$
U(x, \omega)=\frac{x-x^{2}}{2 A(\omega)}, \quad 0 \leq x \leq 1 .
$$

Observe que $U(x, \omega)>0$ para $0<x<1$ e $\omega \in \Omega$. A fim de calcular a função de densidade de probabilidade, $f_{U}(q ; x), q>0$, da solução calcula-se, inicialmente, a função de distribuição cumulativa

$$
\begin{aligned}
F_{U}(q ; x) & =\mathcal{P}(U(x) \leq q)=\mathcal{P}\left(\frac{x-x^{2}}{2 A} \leq q\right)= \\
& =1-\mathcal{P}\left(A \leq \frac{x-x^{2}}{2 q}\right)=1-F_{A}\left(\frac{x-x^{2}}{2 q}\right) .
\end{aligned}
$$

Derivando $F_{U}(q ; x)$ com relação à variável $q$ obtém-se

$$
f_{U}(q ; x)=\frac{d}{d q} F_{U}(q ; x)=\frac{x-x^{2}}{2 q^{2}} f_{A}\left(\frac{x-x^{2}}{2 q}\right)=\frac{\xi(x)}{q^{2}} f_{A}\left(\frac{\xi(x)}{q}\right),
$$

onde $\xi(x)=\left(x-x^{2}\right) / 2$. 


\subsection{Exemplo}

Considere a variável aleatória $A$, uniformemente distribuída no intervalo $[1,2]$, isto é, $f_{A}(q)=1$ quando $1<q<2$ e é nula fora deste intervalo. De (2.2) segue que

$$
f_{U}(q ; x)=\left\{\begin{array}{l}
\frac{\xi(x)}{q^{2}}, \quad \text { se } \frac{\xi(x)}{2}<q<\xi(x), \\
0, \quad \text { caso contrário. }
\end{array}\right.
$$

Observe, para fins de comparação, que a esperança matemática de $U(x)$ é dada por

$$
\begin{aligned}
\mathrm{E}[U(x)] & =\int_{0}^{+\infty} q f_{U}(q ; x) d q=\int_{\xi(x) / 2}^{\xi(x)} q \frac{\xi(x)}{q^{2}} d q= \\
& =\left.\xi(x) \ln (q)\right|_{\substack{\xi(x) \\
\xi(x) / 2}}=\frac{\ln (2)}{2}\left(x-x^{2}\right) .
\end{aligned}
$$

Por outro lado, a solução da equação simplificada (usando $\mathrm{E}[A]=3 / 2$ no lugar de $A$ em (2.1)) é

$$
\tilde{U}(x)=\frac{1}{3}\left(x-x^{2}\right) .
$$

Como $\ln (2) / 2 \approx 0.3466$ as funções dadas em (2.3) e (2.4) não diferem muito para este caso, como mostra a Figura 1(a). A título de ilustração, é apresentada também a média gerada pelo método de Monte Carlo, com 100000 realizações da variável aleatória $A$. Maiores detalhes sobre como se obter a média pelo método de Monte Carlo podem ser encontrados em [8] ou [22].

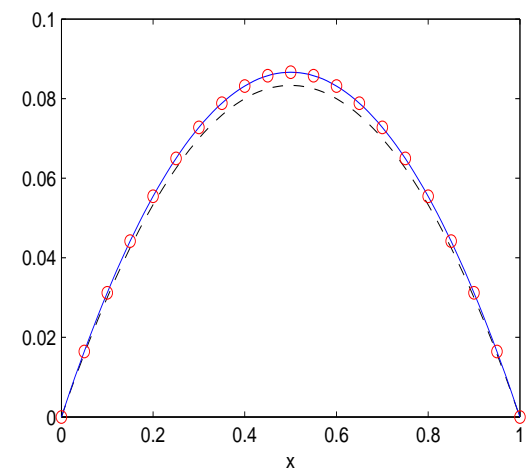

(a)

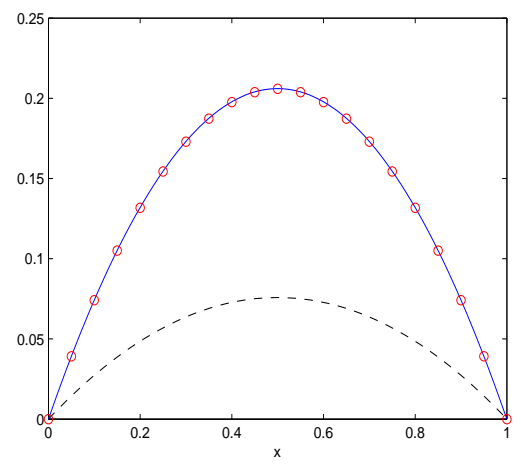

(b)

Figura 1: Ilustração de $\mathrm{E}[U(x)]$ (linha sólida), $\tilde{U}(x)$ (linha pontilhada), e a média da solução obtida pelo método de Monte Carlo (círculos), $0 \leq x \leq 1$. 


\subsection{Exemplo}

Considere agora o caso em que $A$ é lognormal padrão, $A=\exp (\varphi), \varphi \sim N[0,1]$. A função de densidade de probabilidade de $A$ é dada por

$$
f_{A}(q)=\frac{1}{q \sqrt{2 \pi}} \exp \left(-\frac{[\ln (q)]^{2}}{2}\right) .
$$

Tendo em vista $(2.2)$, obtém-se

$$
\begin{aligned}
f_{U}(q ; x) & =\frac{\xi(x)}{q^{2}} f_{A}\left(\frac{\xi(x)}{q}\right)=\frac{\xi(x)}{q^{2}} \frac{1}{\sqrt{2 \pi}[\xi(x) / q]} \exp \left(-\frac{[\ln (\xi(x) / q)]^{2}}{2}\right)= \\
& =\frac{1}{q \sqrt{2 \pi}} \exp \left(-\frac{[\ln (q)-\ln (\xi(x))]^{2}}{2}\right),
\end{aligned}
$$

ou seja, $U(x)$ é também lognormal, $U(x)=\exp (\psi), \psi \sim N[\ln (\xi(x)), 1]$. Usando (2.5) é possível calcular todos os momentos estatísticos de $U(x)$. Em particular, denotando o número de Euler, ou neperiano, por "e", a esperança matemática de $U(x)$ é dada por

$$
\mathrm{E}[U(x)]=\exp \left(\ln (\xi(x))+\frac{1}{2}\right)=\sqrt{\mathrm{e}} \xi(x)=\frac{\sqrt{\mathrm{e}}}{2}\left(x-x^{2}\right) \approx 0.8244\left(x-x^{2}\right),
$$

enquanto a solução da equação simplificada (considerando $\mathrm{E}[A]=\sqrt{\mathrm{e}}$ no lugar de $A$ em (2.1)) é

$$
\tilde{U}(x)=\frac{1}{2 \sqrt{\mathrm{e}}}\left(x-x^{2}\right) \approx 0.3033\left(x-x^{2}\right) .
$$

Na Figura 1(b) é esboçado $\mathrm{E}[U(x)]$ e $\tilde{U}(x)$, bem como a média da solução gerada pelo método de Monte Carlo, com 100000 realizações de $A$. Maiores detalhes sobre momentos estatísticos de uma variável aleatória lognormal podem ser encontrados em [22].

\section{Equação da Advecção Linear Aleatória}

Considere, agora, a equação da advecção linear aleatória

$$
\begin{aligned}
& \frac{\partial}{\partial t} U(x, t)+V \frac{\partial}{\partial x} U(x, t)=0, \quad t>0, \quad x \in \mathbb{R}, \\
& U(x, 0)=U_{0}(x),
\end{aligned}
$$

onde a velocidade, $V$, é uma variável aleatória e a condição inicial, $U_{0}(x)$, é uma função aleatória.

Em [17] é utilizado o método das características [13] e a lei de probabilidade total [15] para mostrar que a função de densidade de probabilidade da solução de (3.1) no ponto $(x, t), f_{U}(q ; x, t)$, é dada por

$$
f_{U}(q ; x, t)=\int_{-\infty}^{\infty} \frac{1}{t} f_{V}\left(\frac{x-x_{0}}{t}\right) f_{U_{0}}\left(q ; x_{0}\right) d x_{0},
$$


ou, equivalentemente,

$$
f_{U}(q ; x, t)=\int_{-\infty}^{\infty} f_{V}(v) f_{U_{0}}(q ; x-v t) d v=\mathrm{E}_{V}\left[f_{U_{0}}(q ; x-V t)\right]
$$

Além disso, no caso em que $U_{0}(x)=u(x)$ é uma função determinística, (3.2) pode ser apresentada como

$$
\begin{aligned}
f_{U}(q ; x, t) & =\int_{-\infty}^{+\infty} f_{V}(v) \delta(q-u(x-v t)) d v= \\
& =\int_{-\infty}^{\infty} \frac{1}{t} f_{V}\left(\frac{x-x_{0}}{t}\right) \delta\left(q-u\left(x_{0}\right)\right) d x_{0},
\end{aligned}
$$

onde $\delta$ é a distribuição delta de Dirac [21].

\subsection{Exemplo}

No caso particular em que $u(x)$ é uma função (determinística) suave, a equação $u(x)-q=0$ tem $\mathrm{n}$ raízes isoladas, $x_{j, q}, j=1,2, \ldots, n$, e a derivada $u^{\prime}(x)$ não se anula em nenhuma das raízes, tem-se (veja [21], por exemplo)

$$
\delta(q-u(x))=\sum_{j=1}^{n} \frac{\delta\left(x-x_{j, q}\right)}{\left|u^{\prime}\left(x_{j, q}\right)\right|}
$$

e, consequentemente,

$$
\begin{aligned}
f_{U}(q ; x, t) & =\int_{-\infty}^{+\infty} \frac{1}{t} f_{V}\left(\frac{x-x_{0}}{t}\right) \sum_{j=1}^{n} \frac{\delta\left(x_{0}-x_{j, q}\right)}{\left|u^{\prime}\left(x_{j, q}\right)\right|} d x_{0}= \\
& =\frac{1}{t} \sum_{j=1}^{n} \frac{1}{\left|u^{\prime}\left(x_{j, q}\right)\right|} f_{V}\left(\frac{x-x_{j, q}}{t}\right) .
\end{aligned}
$$

\subsection{Exemplo}

Considere $V$ Gaussiana, $V \sim N\left(\mu, \sigma^{2}\right)$, e a condição inicial $u(x)$ sendo a função Heaviside,

$$
u(x)= \begin{cases}1, & \text { se } \quad x<0 \\ 0, & \text { se } \quad x>0 .\end{cases}
$$

Esta condição inicial tem sido usada por vários autores para estudar zonas de mistura em concentrações de substâncias [2]. De (3.3), segue que

$$
\begin{aligned}
f_{U}(q ; x, t) & =\delta(q-1) \int_{-\infty}^{x / t} f_{V}(v) d v+\delta(q) \int_{x / t}^{+\infty} f_{V}(v) d v= \\
& =\delta(q)+[\delta(q-1)-\delta(q)] F_{V}\left(\frac{x}{t}\right)
\end{aligned}
$$


isto é, $U(x, t)$ é a variável aleatória discreta de Bernoulli com

$$
\begin{aligned}
\mathcal{P}(U(x, t) & =1)=\int_{-\infty}^{x / t} f_{V}(v) d v=\frac{1}{\sqrt{2 \pi} \sigma} \int_{-\infty}^{x / t} \exp \left[-\frac{(\tau-\mu)^{2}}{2 \sigma^{2}}\right] d \tau= \\
& =\frac{1}{\sqrt{\pi}} \int_{-\infty}^{(x-\mu t) /(\sqrt{2} \sigma t)} \mathrm{e}^{-\tau^{2}} d \tau=1-\frac{1}{2} \operatorname{erfc}\left(\frac{x-\mu t}{\sqrt{2} \sigma t}\right),
\end{aligned}
$$

onde $\operatorname{erfc}(x)$ é a função erro complementar.

Note que $\mathcal{P}(U(x, t)=0)=1-\mathcal{P}(U(x, t)=1)$ e que a esperança da solução é

$$
\mathrm{E}[U(x, t)]=1 \cdot \mathcal{P}(U(x, t)=1)+0 \cdot \mathcal{P}(U(x, t)=0)=1-\frac{1}{2} \operatorname{erfc}\left(\frac{x-\mu t}{\sqrt{2} \sigma t}\right) .
$$

A Figura 2 ilustra a média, $\mathrm{E}[U(x, t)]$, da solução de $(3.1)$ em $t=0.5$ e $t=$ $1.0,-4 \leq x \leq 4$, com $V \sim N(0.5,1)$. Também, apresenta a solução da equação simplificada $\tilde{U}(x, t)=u(x-\mathrm{E}[V] t)=u(x-0.5 t)$, e a solução produzida pelo método de Monte Carlo, com 10000 realizações de $V$. Este é um exemplo de que uma solução simplificada pode omitir propriedades físicas relevantes da solução. A difusão observada na Figura 2, causada pela aleatoriedade de V, é comprovada pelos resultados descritos na sequência (em particular, pelo resultado do Corolário 3.1).

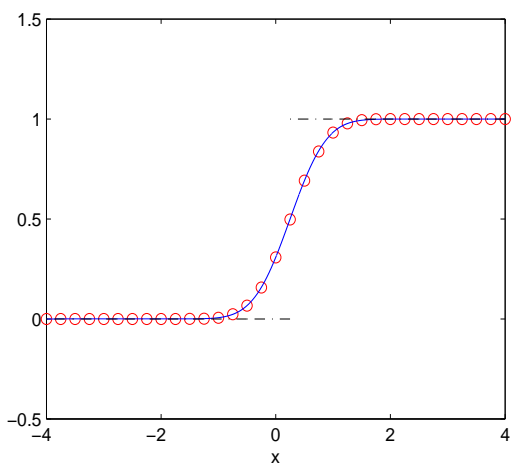

(a)

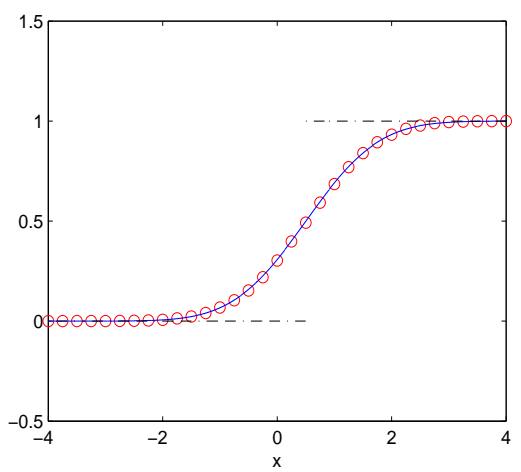

(b)

Figura 2: Ilustração de $\mathrm{E}[U(x, t)]$ (linha sólida), $\tilde{U}(x, t)$ (linha pontilhada), e a média da solução obtida pelo método de Monte Carlo (círculos); $t=0.5$ (a) e $t=1.0(\mathrm{~b})$.

Lema 3.1. No caso em que $V \sim N\left(\mu, \sigma^{2}\right)$, a função $h(x, t ; \zeta)=(1 / t) f_{V}((x-\zeta) / t)$ satisfaz à seguinte equação de advecção-difusão:

$$
\frac{\partial}{\partial t} h(x, t ; \zeta)+\mu \frac{\partial}{\partial x} h(x, t ; \zeta)=\sigma^{2} t \frac{\partial^{2}}{\partial x^{2}} h(x, t ; \zeta)
$$


Demonstração. Como $V \sim N\left(\mu, \sigma^{2}\right)$, segue que sua função de densidade de probabilidade é dada por

$$
f_{V}(x)=\frac{1}{\sqrt{2 \pi} \sigma} \exp \left[-\frac{(x-\mu)^{2}}{2 \sigma^{2}}\right]
$$

com derivadas

$$
\frac{d}{d x} f_{V}(x)=-f_{V}(x)\left[\frac{x-\mu}{\sigma^{2}}\right] \quad \text { e } \quad \frac{d^{2}}{d x^{2}} f_{V}(x)=\frac{f_{V}(x)}{\sigma^{2}}\left[-1+\frac{(x-\mu)^{2}}{\sigma^{2}}\right] .
$$

Deste modo,

$$
\begin{aligned}
\frac{\partial}{\partial t} h(x, t ; \zeta) & =\frac{1}{t^{2}} h(x, t ; \zeta)\left[-t+\frac{(x-\zeta)(x-\zeta-\mu t)}{\sigma^{2} t}\right], \\
\frac{\partial}{\partial x} h(x, t ; \zeta) & =-\frac{1}{t^{2}} h(x, t ; \zeta)\left[\frac{x-\zeta-\mu t}{\sigma^{2}}\right] \mathrm{e} \\
\frac{\partial^{2}}{\partial x^{2}} h(x, t ; \zeta) & =\frac{1}{\sigma^{2} t^{3}} h(x, t ; \zeta)\left[-t+\frac{(x-\zeta-\mu t)^{2}}{\sigma^{2} t}\right] .
\end{aligned}
$$

Portanto,

$$
\begin{aligned}
\frac{\partial}{\partial t} h(x, t ; \zeta) & +\mu \frac{\partial}{\partial x} h(x, t ; \zeta)= \\
& =\frac{1}{t^{2}} h(x, t ; \zeta)\left[-t+\frac{(x-\zeta)(x-\zeta-\mu t)}{\sigma^{2} t}-\frac{\mu t(x-\zeta-\mu t)}{\sigma^{2} t}\right]= \\
& =\frac{1}{t^{2}} h(x, t ; \zeta)\left[-t+\frac{(x-\zeta-\mu t)^{2}}{\sigma^{2} t}\right]=\sigma^{2} t \frac{\partial^{2}}{\partial x^{2}} h(x, t ; \zeta) .
\end{aligned}
$$

Para os Exemplos 3.1. e 3.2., acima, vale o seguinte resultado:

Proposição 3.1. Se $V$ é Gaussiana, $V \sim N\left(\mu, \sigma^{2}\right)$, então as funções de densidade de probabilidade (3.4) e (3.5) satisfazem à seguinte equação de advecção-difusão:

$$
\frac{\partial}{\partial t} f_{U}(q ; x, t)+\mu \frac{\partial}{\partial x} f_{U}(q ; x, t)=\sigma^{2} t \frac{\partial^{2}}{\partial x^{2}} f_{U}(q ; x, t) .
$$

Demonstração. A função de densidade (3.4) pode ser reescrita como

$$
f_{U}(q ; x, t)=\sum_{j=1}^{n} \frac{1}{\left|u^{\prime}\left(x_{j, q}\right)\right|} h\left(x, t ; x_{j, q}\right) .
$$

Tendo em vista que $h\left(x, t ; x_{j, q}\right)$ satisfaz a equação (3.6) para cada $x_{j, q}$, segue que $f_{U}(q ; x, t)$, dada em $(3.4)$, satisfaz (3.7). 
Agora, denotando $\Delta(q)=[\delta(q-1)-\delta(q)]$ e derivando (3.5), obtém-se

$$
\begin{aligned}
\frac{\partial}{\partial t} f_{U}(q ; x, t) & =\Delta(q) f_{V}\left(\frac{x}{t}\right)\left(-\frac{x}{t^{2}}\right)=\Delta(q) h(x, t ; 0)\left(-\frac{x}{t}\right) \\
\frac{\partial}{\partial x} f_{U}(q ; x, t) & =\Delta(q) f_{V}\left(\frac{x}{t}\right)\left(\frac{1}{t}\right)=\Delta(q) h(x, t ; 0), \quad \mathrm{e} \\
\frac{\partial^{2}}{\partial x^{2}} f_{U}(q ; x, t) & =\Delta(q) f_{V}^{\prime}\left(\frac{x}{t}\right)\left(\frac{1}{t^{2}}\right)=-\Delta(q) f_{V}\left(\frac{x}{t}\right)\left(\frac{x / t-\mu}{\sigma^{2}}\right)\left(\frac{1}{t^{2}}\right)= \\
& =-\Delta(q) h(x, t ; 0)\left(\frac{x / t-\mu}{\sigma^{2} t}\right) .
\end{aligned}
$$

Assim,

$$
\frac{\partial}{\partial t} f_{U}(q ; x, t)+\mu \frac{\partial}{\partial x} f_{U}(q ; x, t)-\sigma^{2} t \frac{\partial^{2}}{\partial x^{2}} f_{U}(q ; x, t)=0,
$$

ou seja, (3.5) satisfaz (3.7).

Corolário 3.1. As médias, $\mathrm{E}[U(x, t)]$, das soluções dos problemas dos Exemplos 3.1. e 3.2., com $V \sim N\left(\mu, \sigma^{2}\right)$, satisfazem à equação de advecção-difusão

$$
\frac{\partial}{\partial t} \mathrm{E}[U(x, t)]+\mu \frac{\partial}{\partial x} \mathrm{E}[U(x, t)]=\sigma^{2} t \frac{\partial^{2}}{\partial x^{2}} \mathrm{E}[U(x, t)]
$$

Observação 3.1. Os resultados da Proposição 3.1 e do Corolário 3.1 continuam válidos para a solução de (3.1), com $V \sim N\left(\mu, \sigma^{2}\right)$, desde que a derivação, em $x$ e t, sob o sinal de integração em (3.2) seja possível. Para tanto, são necessárias hipóteses de regularidade sobre a função $f_{U_{0}}(q ; x)$ (veja [12], p. 268, por exemplo).

\section{Problema de Burgers-Riemann Aleatório}

Considere o caso não-linear obtido pelo problema de Riemann aleatório para a equação de Burgers (introduzida por J. M. Burgers em [3])

$$
\begin{aligned}
& \frac{\partial}{\partial t} U(x, t)+\frac{1}{2} \frac{\partial}{\partial x} U^{2}(x, t)=0, \quad t>0, \quad x \in \mathbb{R}, \\
& U(x, 0)=\left\{\begin{array}{lll}
U_{L}, & \text { se } \quad x<0 \\
U_{R}, & \text { se } & x>0
\end{array}\right.
\end{aligned}
$$

em que os estados iniciais, $U_{L}$ e $U_{R}$, são variáveis aleatórias. Observe que aqui a aleatoriedade aparece apenas na condição inicial, transformando o problema como um todo numa equação diferencial estocástica. Para uma simples realização $U_{L}(\omega)$ e $U_{R}(\omega)$, de $U_{L}$ e $U_{R}$, respectivamente, tem-se o problema de Burgers-Riemann determinístico:

$$
\begin{aligned}
& \frac{\partial}{\partial t} u(x, t, \omega)+\frac{1}{2} \frac{\partial}{\partial x} u^{2}(x, t, \omega)=0, \quad t>0, \quad x \in \mathbb{R}, \\
& u(x, 0, \omega)=\left\{\begin{array}{lll}
U_{L}(\omega), & \text { se } \quad x<0, \\
U_{R}(\omega), & \text { se } \quad x>0 .
\end{array}\right.
\end{aligned}
$$


Soluções fisicamente corretas de (4.2), isto é, soluções de entropia, são ondas de rarefação ou ondas de choque [13]. Em [5] é mostrado que a solução de (4.1), em $(x, t)$, pode ser representada por

$$
U(x, t)=U_{L} \mathcal{X}_{\mathcal{R}^{-}}+\beta \mathcal{X}_{\mathcal{R}^{0}}+U_{R} \mathcal{X}_{\mathcal{R}^{+}},
$$

onde $\beta=x / t$, e $\mathcal{X}_{\mathcal{R}^{-}}, \mathcal{X}_{\mathcal{R}^{0}}$ e $\mathcal{X}_{\mathcal{R}^{+}}$são as funções características dos conjuntos mutuamente exclusivos definidos na Figura 3. Observe que a função característica de $\mathcal{R}^{0}$, por exemplo, é definida por:

$$
\mathcal{X}_{\mathcal{R}^{0}}=\left\{\begin{array}{lll}
1, & \text { se } & \left(U_{L}, U_{R}\right) \in \mathcal{R}^{0}, \\
0, & \text { se } & \left(U_{L}, U_{R}\right) \notin \mathcal{R}^{0} .
\end{array}\right.
$$

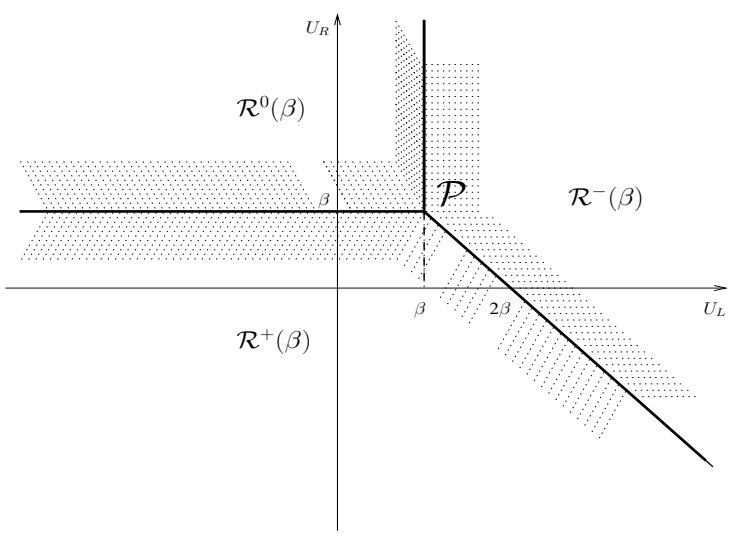

Figura 3: Ilustração das regiões de integração.

O m-ésimo momento estatístico de $U(x, t)$ é dado por

$$
\begin{aligned}
& \mathrm{E}\left[U^{m}(x, t)\right]=\iint_{\mathcal{R}^{-}} u_{L}^{m} f_{U_{L} U_{R}}\left(u_{L}, u_{R}\right) d u_{L} d u_{R}+ \\
& \quad+\beta^{m} \iint_{\mathcal{R}^{0}} f_{U_{L} U_{R}}\left(u_{L}, u_{R}\right) d u_{L} d u_{R}+\iint_{\mathcal{R}^{+}} u_{R}^{m} f_{U_{L} U_{R}}\left(u_{L}, u_{R}\right) d u_{L} d u_{R} .
\end{aligned}
$$

Para a obtenção dos momentos estatísticos da solução de (4.1), usando a expressão acima, deve-se calcular três integrais duplas para cada valor de $\beta$. Em alguns casos é possível calcular tais integrais exatamente, como ilustrado no exemplo a seguir.

\subsection{Exemplo}

Considere o caso em que $U_{L}$ e $U_{R}$ são variáveis aleatórias independentes e uniformemente distribuídas no intervalo $[-a, a]$. Alguns cálculos mostram que a média da solução de (4.1) é dada por

$$
\mathrm{E}[U(x, t)]= \begin{cases}-\frac{\beta}{4 a^{2}}(\operatorname{sgn}(\beta) \beta-a)^{2}, & \text { se }-a \leq \beta \leq a, \\ 0, & \text { caso contrário, }\end{cases}
$$


$\operatorname{com} \beta=x / t \mathrm{e}$

$$
\operatorname{sgn}(\beta)=\left\{\begin{array}{cl}
-1, & \text { se } \beta<0, \\
0, & \text { se } \beta=0, \\
1, & \text { se } \beta>0 .
\end{array}\right.
$$

Na Figura 4 é ilustrada a média, $\mathrm{E}[U(x, t)]$, da solução de (4.1) em $t=0.4$ e $t=0.8,-1 \leq x \leq 1$. $U_{L}$ e $U_{R}$ são independentes e uniformemente distribuídas em $[-1,1]$. Convém ressaltar que a solução da equação simplificada de (4.1), com $\mathrm{E}\left[U_{L}\right]=\mathrm{E}\left[U_{R}\right]=0$ no lugar de $U_{L}$ e $U_{R}$, é identicamente nula (linha tracejada na Figura 4).

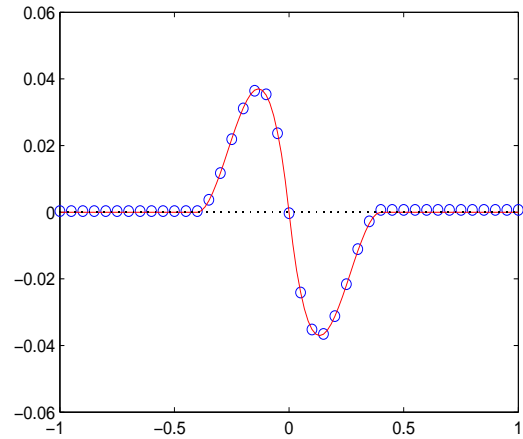

(a)

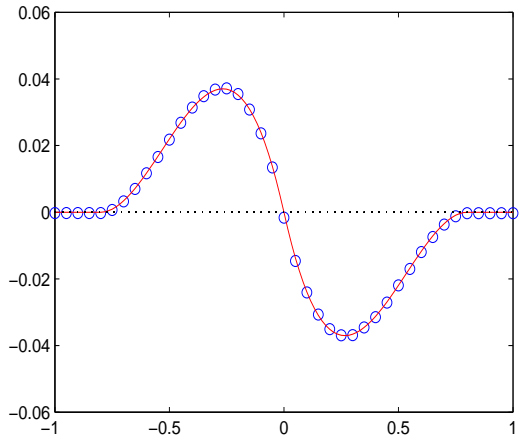

(b)

Figura 4: Ilustração da média, $\mathrm{E}[U(x, t)]$, dada por (4.3) (linha sólida) e da média obtida por Monte Carlo (círculos), $t=0.4$ (a) e $t=0.8$ (b), $-1 \leq x \leq 1$.

\section{Conclusões}

Este trabalho apresentou alguns exemplos de soluções de equações diferenciais com dados aleatórios, comparando suas médias com as soluções de equações diferenciais determinísticas resultantes da substituição dos parâmetros aleatórios por suas médias.

Os exemplos ilustram que a substituição de parâmetros aleatórios por suas médias leva a previsões qualitativamente errôneas da média da solução das equações (Figuras 1(b), 2 e 4). Por outro lado, a obtenção da função de densidade de probabilidade da solução por meio da função de distribuição cumulativa levou não apenas ao cálculo de médias da solução consistentes com o método de Monte Carlo, como também garantiu informações estatísticas completas sobre a solução.

Este trabalho, complementado com as referências citadas abaixo, pode servir como ponto de partida para pesquisadores interessados em substituir custosas téc- 
nicas de simulação por representações analíticas dos momentos estatísticos procurados.

\section{Agradecimentos}

Os autores agradecem o apoio financeiro do CNPq (Processos 303701/2010-2 e 314553/2009-6) e aos revisores pelas diversas sugestões.

Abstract. We study through some examples the influence of uncertainties on the solution of differential equations with random initial data and/or random parameters. These examples show that the mean of the solution, which is usually a relevant information on applications, may be qualitatively different from the approximate solution computed from a deterministic differential equation in which the random parameters are replaced by their averages.

\section{Referências}

[1] J.S. Azevedo, S.P. Oliveira, O. A. L. Lima, Métodos estocásticos para modelagem de escoamento estacionário e transiente em meios porosos. Rev. Bras. Geof., 27 (2009), 241-254.

[2] M.R. Borges, F. Furtado, F. Pereira, H.P.A. Souto, Scaling analysis for the tracer flow problem in self-similar permeability fields. Multiscale Model. Simul., 7 (2008), 1130-1147.

[3] J.M. Burgers, A mathematical model illustrating the theory of turbulance. Ad. Appl. Mech., 1 (1948), 171-179.

[4] P.L. Chow, "Stochastic Partial Differential Equations", Chapman \& Hall/CRC, New York, 2007.

[5] M.C.C. Cunha, F.A. Dorini, Statistical moments of the solution of the random Burgers-Riemann problem. Math. Comput. Simulation, 79 (2009), 1440-1451.

[6] F.A. Dorini, M.C.C. Cunha, Soluções de problemas envolvendo equações diferenciais em que existem incertezas, em "Anais do XXXIII Congresso Nacional de Matemática Aplicada e Computacional", Águas de Lindóia, SP, 3 (2010), 180-186.

[7] R. Durrett, "Stochastic Calculus: a practical introduction", CRC Press, New York, 1996.

[8] G.S. Fishman, "Monte Carlo: concepts, algorithms and applications", SpringerVerlag, New York, 1996.

[9] D. Gottlieb, D. Xiu, Galerkin method for wave equations with uncertain coefficients. Commun. Comput. Phys., 3 (2008), 505-518.

[10] I. Karatzas, S.E. Shreve, "Brownian Motion and Stochastic Calculus", SpringerVerlag, New York, 1988. 
[11] V. Klyatskin, "Stochastic Equations and Waves in Randomly Inhomogeneous Media", Nauka, Moscou, 1980.

[12] T.W. Korner, "Fourier Analysis", Cambridge University Press, Cambridge, 1988.

[13] R.J. LeVeque, "Numerical Methods for Conservation Laws", Birkhäuser, Berlin, 1992.

[14] B.K. Oksendal, "Stochastic Differential Equations: an introduction with applications", Springer, New York, 2000.

[15] A. Papoulis, "Probability, Random Variables, and Stochastic Processes", McGraw-Hill, Inc., New York, 1984.

[16] S.B. Pope, Lagrangian PDF methods for turbulent flows. Annu. Rev. Fluid Mech., 26 (1994), 23-63.

[17] L.T. Santos, F.A. Dorini, M.C.C. Cunha, The probability density function to the random linear transport equation. Appl. Math. Comput., 216 (2010), $1524-1530$.

[18] M. Shvidler, K. Karasaki, Exact averaging of stochastic equations for transport in random velocity field. Transp. Porous Media, 50 (2003), 223-241.

[19] T.T. Soong, "Random Differential Equations in Sciences and Engineering", Academic Press, New York, 1973.

[20] D. Xiu, "Numerical Methods for Stochastic Computations: a spectral method approach", Princeton University Press, Princeton, 2010.

[21] E. Zauderer, "Partial Differential Equations of Applied Mathematics", John Wiley \& Sons, New York, 1983.

[22] D. Zhang, "Stochastic Methods for Flow in Porous Media - Coping with Uncertainties", Academic Press, San Diego, 2002. 